\title{
ESPERTO: UM SISTEMA INTEGRADO PARA MICROANÁLISES EM SONDAS ELETRÔNICAS MANUAIS A TRÊS ESPECTRÔMETROS
}

\section{RESUMO}

ESPERTO é um sistema integrado desenvolvido para aquisição "on line" e tratamento de dados de quimismo de materiais através de microanálise com Sondas Eletrônicas manuais providas de três espectrômetros. Inclue diversos programas escritos em linguagem BASIC e arquivos de dados que monitoram todo o procedimento analítico adotado no laboratório de Microssonda Eletrônica do Departamento de Mineralogia e Petrologia do IG/USP, que inclue etapas de aquisição de dados, de correção para fatores experimentais (radiação de fundo, deriva e tempomorto dos contadores) e correções empíricas ("correção beta") para os efeitos de matriz (número atômico, absorção de massa e fluorescência secundária).

\section{ABSTRACT}

An integrated software system, ESPERTO, has been developed for on-line acquisition and treatment of chemical data through microanalysis of materials by manual electron microprobe equiped with three spectrometers. The system comprises various programs written in BASIC and data archives that monitor the entire routine analytical procedure employed in the Electron Microprobe Laboratory of the Mineralogy and Petrology Department of the Geosciences Institute of the São Paulo University, which includes the following steps: collection of data, correction for experimental

'Departamento de Mineralogia e Petrologia, Instituto de Geociências/USP, São Paulo. 
factors (background radiation, drift and dead-time of the counters) and empirical corrections ("beta correction") for matrix effects (atomic number, mass absorption, and secondary fluorescence).

\section{INTRODUÇAOO}

Um dos principals problemas associados com o procedimento microanalitico para a determinação de composiçăo química de materiais, com a utillzação da sonda eletrônica manual do Laboratório de Microssonda Eletrônica do Departamento de Mineralogia e Petrologla do IG/USP. reside no fato de que as etapas necessárias de correçōes $\theta$ conversões dos dados adquiridos durante as rotinas analiticas săo multo laboriosas e dispendem parcela excessiva de tempo, uma vez que săo realizadas quer manualmente, quer com auxilio de computadores de grande porte não diretamente conectados.

A utillzaçăo de microcomputadores traz grandes simplificaçőes e torna multo mais ágil todo o procedimento, eliminando, por exemplo, etapas exaustivas de digitaçăo de dados parciais, $\theta$ apresentando a vantagem adicional de permitir tratamentos parcials ou completos de um conjunto de dados imediatamente após a sua aquisição, o que leva, naturalmente, ao controle mais adequado das condições analitico-experimentais. ESPERTO é um sistema integrado, simples, em sua maior parte desenvolvido no referido Laboratório, destinado a minimizar tais deficiências. Consiste de diversos programas e arquivos de dados estruturados em obediência à sistemática analfica adotada já há vários anos pelos usuários do Laboratório, e, de modo a possibilitar diversas interferências oportunas e também controlar situações de erro inadvertidamente provocadas. Fol concebido para Sondas equipadas com três espectrômetros, mas pode ser rapidamente adaptado para configuraçöes distintas.

O conjunto apresentado de programas monitora desde a aquisiçăo "on line" de dados, traduzidos por intensidade de radiação medida em cada espectrômetro e respectiva corrente de análise, medida no felxe eletrônico (beam current) ou na amostra (sample current), por intervalo selecionado de tempo, até o cálculo dos resultados finais, expressos em porcentagem em peso de óxidos, número de cátions, etc., por comparação com materiais-padrão (em geral minerais vários). Etapas intermediárias incluem correções devidas a fatores experimentais e efeitos de matriz.

\section{PROCEDIMENTO GERAL E ESTRUTURA DE ESPERTO}


Em essência, a sistemática rotineira do Laboratório (Fig. 1) comporta três etapas distintas, consecutivas, representadas, respectivamente, pela aquisição de dados (1) relativos a medidas de radiação de fundo (background), medidas para avaliações de deriva (drift) e medidas de materiais-problema (amostras) e materiais-padrão para os elementos químicos previamente sintonizados nos espectrômetros (no caso, cada rotina possibilita análise para três elementos) e sua armazenagem. $\mathrm{Na}$ fase seguinte (2), estes dados são corrigidos para os fatores experimentais, quais sejam, deriva, radiação de fundo e, opcionalmente, tempo-morto (dead-time) dos contadores e convertidos para porcentagem em peso de óxidos por comparação com os padrões medidos, em uma primeira aproximação composicional; estes resultados (parciais) são também armazenados. Este procedimento é repetido até que se tenham os dados corrigidos em primeira aproximação para todos os elementos que se deseja analisar em uma determinada amostra; após (3), são conjuntamente corrigidos para os efeitos de matriz, dominados pelos fatores de número atômico, absorção de massa e fluorescência secundária (v. detalhes em KEIL, 1967; HEINRICH, 1981; GOLSDTEIN et al.,1981 e GOMES, 1984 entre outros).

O conjunto ESPERTO contém quatro programas e cinqüenta $\theta$ oito arquivos de dados essenciais; dois programas e um arquivo adicionais prestam-se à iniciação e verificação do sistema e à interligação entre os demais. As relações entre programas e com os periféricos são diagramadas na Figura 2 (v. também VLACH, 1988). Abaixo, são listados os programas, arquivos, e as respectivas funções:

.HEนO, iniciação e verificação do sistema;

.ESPERTO, "menu" de interligação entre os programas e com o sistema;

.ANLS ROTINA, aquisição "on line" de dados (iniciais), listagens e arquivamento;

.CORR DRIFT1, correção dos dados iniciais para os fatores experimentais, conversão para quantidades em óxidos em primeira aproximação, listagens e arquivamento;

.CORREC BETA, correção de um conjunto de resultados obtidos em primeira aproximação para efeitos de matriz, de acordo com o método preconizado por BENCE e ALBEE (1968) e conversões para fómulas diversas;

.REORG ARQUI, manutenção, correção e criação de arquivos de dados (iniciais ou resultados de primeira aproximação composicional);

.OX-01 a OX-58, dados internos (dados gerais e fatores utilizados pelo programa CORREC BETA, segundo ALBEE \& RAY, 1970;

.PICTURE, tela gráfica com um logotipo. 

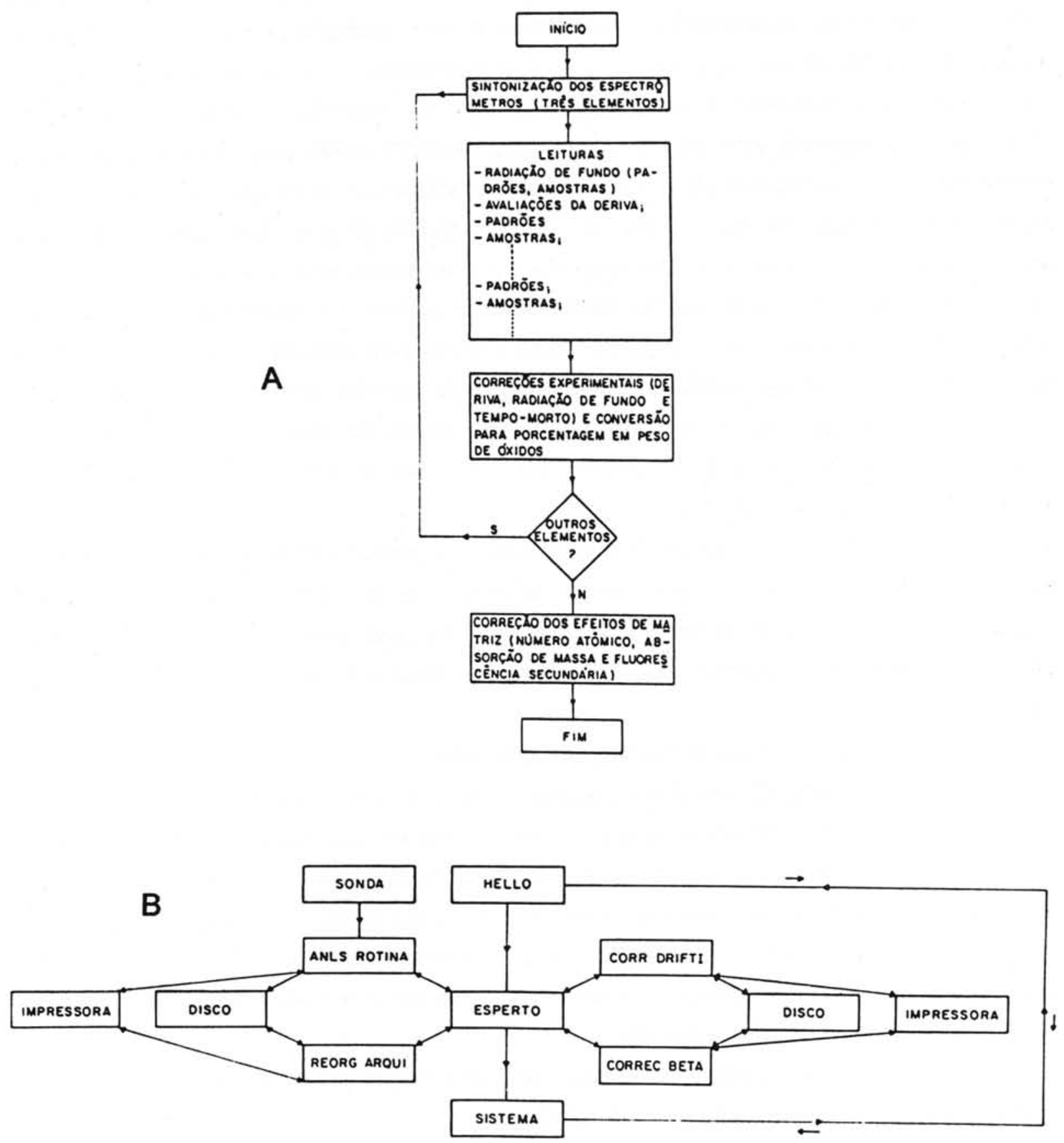

Figura 1 - Fluxogramas. A. Procedimento analítico rotineiro para microanálises no Laboratório de Microssonda Eletrônica do Departamento de Mineralogia e Petrologia. B: interrelaçăo entre programas e com periféricos para o "software" ESPERTO (< indica aborto de procedimento). 


\section{DESCRIÇÃO DOS PROGRAMAS}

Todos os programas apresentados são escritos em linguagem BASIC simples, para microcomputadores de 8 bits compativeis com a linha APPLE, e de utilização relativamente fácil, com pedidos de entradas e "menus" em geral auto-explicativos; rotinas para contorno das principais situaçöes de erro previnem a maioria dos problemas que possam acarretar perda parcial ou total de dados em aquisição e/ou tratamento e informam ao usuário o erro em que este incorreu.

Os arquivos com dados internos (OX-01 a OX-58) são seqüenciais; já os dados analfticos (iniciais e parciais) são armazenados em modo aleatório para facilitar o seu manuseio, otimizar as rotinas de pesquisa e permitir a flexibilidade necessária no comprimento dos registros em função do número de elementos que se pretenda analisar. Os resultados finals (após a correção dos efeitos de matriz) são descarregados diretamente em impressora.

Cada um dos programas é discutido brevemente a seguir; maiores especificações são detalhadas por VLACH (1988).

\section{INICIAÇÃO: OS PROGRAMAS HEUO E ESPERTO}

Tudo o que o programa HELO faz é iniciar o sistema e, após rápida verificação dos programas e arquivos, carregar por alguns instantes uma tela gráfica com um logotipo (armazenado em PICTURE) e, a seguir, o programa ESPERTO, o qual apresenta um "menu" que permite ao usuário selecionar o programa desejado ou retornar ao sistema (v. Fig. 2). Em caso de problemas com os programas, arquivos ou com sistema, o procedimento é abortado.

\section{AQUISIÇÃO DE DADOS: O PROGRAMA ANLS ROTINA}

Este programa é responsável pela aquisição de dados de intensidade de radiação, expressa em número de contagens medida em cada espectrômetro e respectivas correntes (feixe/amostra), em $\eta A$, por intervalo selecionado de tempo. Ao ser carregado, pede ao usuário informaçőes genéricas (e.g., ttulos, data) e de tipo analttico, tais como elementos sintonizados nos espectrômetros, intervalo de tempo de integração dos impulsos nos contadores, número de leituras a efetuar por "ponto" (volume) analisado, etc. A seguir, ingressa em um estágio de espera de 
comandos, os quais podem ser de dois tipos distintos: de aquisição e de operação.

\section{Comandos de Aquisição}

São os que permitem acesso à interface microcomputador-Sonda, possibilitando a entrada de dados. São três em número: BCK, PPD e QUALQUER, que informam, respectivamente, que serão recebldos dados referentes a leituras de radiação de fundo (para padrões e/ou amostras) e de leituras de "pico" para padrōes e amostra genérica, intitulada QUALQUER; nos dois primeiros casos, devem ser fornecidos tftulos especfficos para os padröes/amostras em leitura, imediatamente após os comandos BCK $\Theta$ PPD.

Os dados progressivamente recebidos săo discriminados por espectrómetro em vídeo e devem ser aceitos ou rejeitados pelo operador. No caso de medidas de radlações de fundo $\theta$ padrões såo efetuadas sempre cinco leituras por "ponto"; já para amostras, este número pode variar entre um a cinco. $O$ máximo de dados que pode permanecer simultaneamente em memória corresponde a um total de aproximadamente 300 leituras pontuais.

\section{Comandos de Operação}

Estes passam o controle para rotinas que realizam tarefas especfficas, não relacionadas com a aquisição de dados. Podem ser acionados a qualquer tempo no decorrer de uma rotina de análise. São igualmente em número de três, com as seguintes chamadas e funções:

.CCR, chama rotina para tratamento estatistico (regressão linear e correlação) $\theta$ "display" gráfico para as variaçöes de número de contagens obtidas em função da corrente (feixe/amostra). É utilizada, mais freqüentemente, para avaliações de deriva, com vistas às correções experimentais;

.LA, chama rotina para listagens (em vídeo e/ou impressora) dos dados em memória e seu arquivamento. As opçöes de arquivamento incluem abertura de novos arquivos e adição de dados a arquivos já existentes, como blocos distintos ou não;

.RCO, chama rotina para correções e/ou atualizações das especificações gerais e/ou analficas; 
O programa CORR DRIFT1 tem como funçăo corrigir dados iniclais, armazenados em arquivos criados com ANLS ROTINA, e convertêtos para porcentagem em peso de óxidos, em primeira aproximação, através de comparação com os valores fornecidos para os padrões simultaneamente analisados.

A correção para o tempo-morto $(\tau)$ dos contadores é efetuada através da seguinte funçăo, aplicável para valores de $\tau$ inferiores a $10 \mu$ s (KEIL, 1967):

$$
c_{r}=C_{0} /\left(1-C_{0} \cdot \tau\right)
$$

em que:

$$
\begin{aligned}
& \mathrm{C}_{\mathrm{r}}=\text { número real de contagens; } \\
& \mathrm{C}_{\mathrm{O}}=\text { número observado de contagens; } \\
& \tau=\text { tempo-morto (em s). }
\end{aligned}
$$

Nos trabalhos analiticos rotineiros, este fator não é significativo, uma vez que as contagens obtidas por espectrômetro se situam, em geral, abalxo de $5.10^{3}$ cont/s, valor médio acima do qual esta correção é relevante. Métodos para a avaliação do tempo-morto de contadores são discutidos por KEIL (1967) entre outros.

Para as correções devidas à deriva e radlação de fundo bem como conversões dos dados de contagens em porcentagens em peso de óxidos estão disponfveis quatro opções distintas, entre as quais o usuário deverá selecionar a que melhor se aplica, considerando o procedimento analftico adotado e as condiçőes analfico-instrumentais. As equações de correção e concomitante conversão para óxidos empregadas são as seguintes:

$$
\begin{aligned}
& O X_{a}=O X_{p} \cdot \frac{C_{a}-C_{B a} \cdot\left(I_{a} / I_{B a}\right)}{C_{p}-C_{B p} \cdot\left(I_{a} / I_{B p}\right)} \\
& O X_{a}=O X_{p} \cdot \frac{\left(C_{a}-C_{B a} \cdot\left(I_{a} / I_{B a}\right)\right) \cdot\left(I_{p} / I_{a}\right)}{\left(C_{p}+F\right)-C_{B p} \cdot\left(I_{p} / I_{B p}\right)}
\end{aligned}
$$

nas quais:

OX = porcentagem em peso de 6 xidos; 


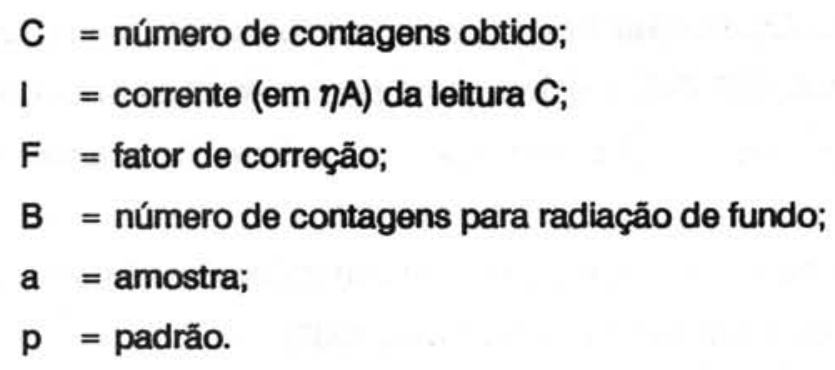

As duas primeiras opções de correção utilizam a equação (2); na primeira, as contagens para os padrões são obtidas por interpolação linear (Equação $4 \mathrm{com} E=0$ ) a partir dos coeficientes obtidos por regressão linear (opção CCR do programa ANLS ROTINA). A opção dois considera, adicionalmente, um fator empírico E, que é reavaliado a cada nova leitura de padrões registrada nos arquivos (v. Fig. 3a) e considerado sempre que os resultados sucessivos mostrem uma divergência superior a $1 \%$. Ou seja:

$$
C_{p}=Y a \cdot I+Y b+E
$$

em que:

$\mathrm{C}_{\mathrm{p}}=$ número de contagens interpolado para o padrão;

$\mathrm{Ya}, \mathrm{Yb}=$ coeficientes de regressão linear;

I = corrente (em $\eta A$, amostra/feixe) de leitura da amostra em correção;

$\mathrm{E}$ = fator empírico.

$\mathrm{Na}$ terceira opção, procura-se, simplesmente, entre os dados de padrões disponfiveis em arquivo, aquele cuja corrente de leitura é a mais próxima da corrente de leitura obtida para a amostra que está em correção e se aplica a equação (3), com F=0 (Fig. 3b). Na opção final, a deriva é avaliada tomando-se as contagens e respectivas correntes de leitura para duas leituras sucessivas registradas de padrão e comparando-se os resultados medidos com os que seriam esperados por dependência linear (Fig. 3c). A diferença obtida é transformada em acréscimos/decréscimos progressivos (fator $F$ na equação 3), de tal forma que:

$$
\mathrm{F}=\mathrm{m} \cdot \frac{\Delta}{\mathrm{n}}
$$

em que:

$\mathrm{F}$ = fator de correção para as contagens dos padrões; 
padrões);

$\Delta=$ deriva total (diferença entre as contagens obtidas e esperadas para os

$m$ = número de leituras de amostras entre os padrões considerados;

$\mathrm{n}$ = número da leitura da amostra em correção, após o primeiro padrão

considerado;

As opções (1) e (3) prestam-se para rotinas analíticas de curta duração e para condições de grande estabilidade do aparelho; para rotinas mais longas e/ou condições não tão ideais de estabilidade devem ser utilizadas preferencialmente as opções (2) e (4), que realizam correções mais rigidas para a deriva. As opções (3) e (4) têm a vantagem da não necessidade do trabalho inicial com a opção CCR do programa ANLS ROTINA, porém implicam na obrigatoriedade de leituras de padrões intercaladas com as leituras de amostras, o que não ocorre com opções (1) e (2).

Em seu início, o programa pede informações relativas ao tftulo, número de blocos de dados do arquivo-fonte e título(s) da(s) amostra(s) para corrigir. A seguir, para cada um dos blocos e, dentro destes, para cada elemento analisado, deve-se selecionar, entre os padrões disponiveis, o que se pretende utilizar e informar a respectiva porcentagem em peso do óxido bem como se a correção para tempo-morto deve ser incluída (em caso afirmativo são pedidos os valores para cada canal). O procedimento de correção é realizado em separado para cada elemento.

Os resultados obtidos (primeira aproximação da porcentagem em peso dos óxidos) são listados e armazenados. As opções para arquivamento permitem abrir um novo arquivo (1); acrescentar dados verticalmente (2) ou horizontalmente (3) a arquivos já existentes. Estas duas últimas opções destinam-se à adição de dados corrigidos a partir de diferentes arquivos-fonte no mesmo arquivo de dados parciais e a adição de dados parciais para até 16 óxidos por amostras, obtidos através de etapas analfticas diversas. Estes dados parciais (primeira aproximação) alimentam o programa CORREC BETA, descrito a seguir.

\section{CORREÇÃO dOS EFEITOS DE MATRIZ: O PROGRAMA CORREC BETA}

Este programa é uma adaptação do programa BETA (versão "mainframe" de H.ULBRICH (inédita), previamente reescrita para BASIC por I.SONOKI (CPGeo-USP). As alterações ora introduzidas estão, em sua maioria, relacionadas aos mecanismos de entrada de dados, de modo a torná-los mais simples e auto-explicativos e permitir a manipulação com arquivos de dados 

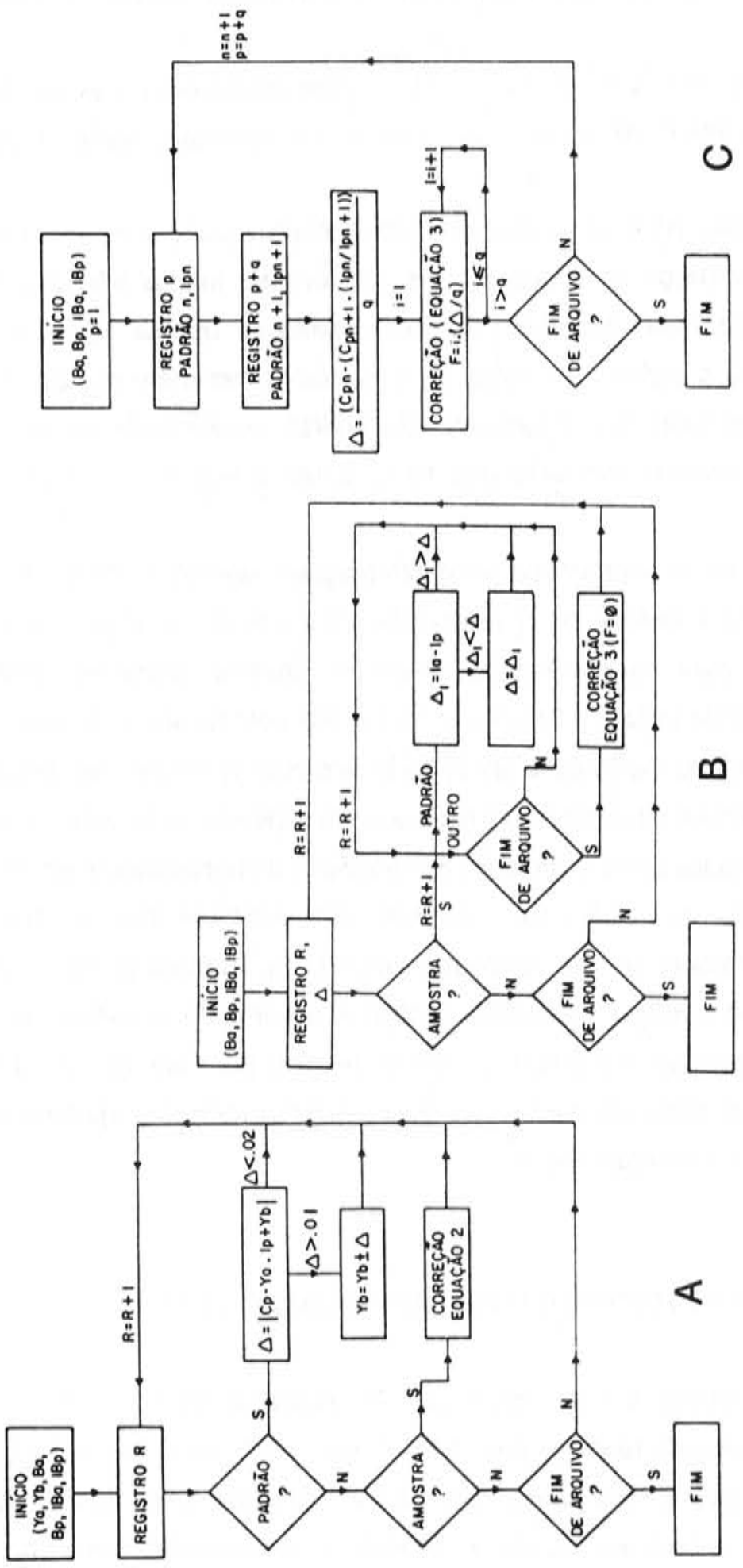

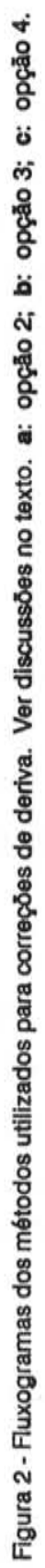


analfticos.

O programa efetua as correções devidas ao número atômico, à absorção de massa e à fluorescência secundária para um conjunto de óxidos (máximo de 16), quantitativamente expressos em frações em peso, em que a soma destas frações é superior a 0,8 e inferior a 1,2, de acordo com o método empírico de "correção beta" desenvolvido por BENCE \& ALBEE (1968) (v. também ZIEBOLD \& OGILVIE, 1964; KEIL, 1967; HEIN RICH, 1981 e GOLDSTEIN et all., 1981).

Nos arquivos OX-01 a OX-58, além de dados gerais (tftulos, número de cátions e oxigênio por fórmula-óxido, etc.), estão armazenados os fatores $\alpha$ calculados a partir de curvas de calibração para sistemas multicomponentais por ALBEE \& RAY (1970), para cinqüenta e oito óxidos para os quais o método se aplica (Tabela 1). Estes são utilizados, juntamente com as frações em peso, para o cálculo dos fatores $\beta$ (equação 6 e seguintes).

CORREC BETA apresenta três opções destinadas, respectivamente, ao cálculo dos fatores $\beta$ para um conjunto de óxidos com análises definitivas, como é o caso de padrões (1); para correção preliminar de um conjunto de óxidos (avaliados em primeira aproximação) para os quais não estão disponfveis fatores $\beta$ de padrões (2) e correção final para um conjunto de dados (de óxidos avaliados em primeira aproximação), em que os fatores $\beta$ dos padrões utilizados são fornecidos.

Para o cálculo dos fatores $\beta$ é utilizada a seguinte função, rearranjada de BENCE \& \& ALBEE (1968):

$$
\text { a. } \quad \beta_{i, j}=\frac{\sum_{j=1}^{n} \omega_{j} \cdot \alpha_{j}^{i}}{\sum_{j=1}^{n} \omega}
$$

em que:

$$
\begin{aligned}
& \beta_{\mathrm{ij}}=\text { fator } \beta \text { do óxido } \mathrm{i} \text { em mistura de } \mathrm{n} \text { óxidos; } \\
& \omega_{\mathrm{j}}=\text { fração em peso do óxido j na mistura; } \\
& \alpha_{\mathrm{j}}=\text { fator } \alpha \text { para o binário } \mathrm{i}, \mathrm{j} \text {. }
\end{aligned}
$$

No procedimento rotineiro do Laboratório, a opção (1) é utilizada para o cálculo dos fatores $\beta$ para os padrões lá disponiveis; tais valores encontram-se em sua maioria já calculados e tabulados. 
Tabela 1 - Óxidos para os quais se aplica o programa CORREC BETA para correção dos efeitos de matriz (máximo de 16 simultâneos).

$\begin{array}{lllllll}\mathrm{H}_{2} \mathrm{O} & \mathrm{LO}_{2} & \mathrm{BeO} & \mathrm{B}_{2} \mathrm{O}_{3} & \mathrm{CO}_{2} & \mathrm{~N}_{2} \mathrm{O}_{5} & \mathrm{~F} \\ \mathrm{NaO} & \mathrm{MgO} & \mathrm{Al}_{2} \mathrm{O}_{3} & \mathrm{SiO}_{2} & \mathrm{P}_{2} \mathrm{O}_{5} & \mathrm{SO}_{3} & \mathrm{Cl} \\ \mathrm{K} & \mathrm{CaO} & \mathrm{Sc}_{2} \mathrm{O}_{3} & \mathrm{TiO}_{2} & \mathrm{~V}_{2} \mathrm{O}_{3} & \mathrm{Cr}_{2} \mathrm{O}_{3} & \mathrm{MnO} \\ \mathrm{FeO} & \mathrm{CoO} & \mathrm{NiO} & \mathrm{CuO} & \mathrm{ZnO} & \mathrm{Ga}_{2} \mathrm{O}_{3} & \mathrm{GeO}_{2} \\ \mathrm{Rb}_{2} \mathrm{O} & \mathrm{SrO} & \mathrm{Y}_{2} \mathrm{O}_{3} & \mathrm{ZrO}_{2} & \mathrm{Nb}_{2} \mathrm{O}_{5} & \mathrm{MoO}_{3} & \mathrm{SnO}_{2} \\ \mathrm{CsO}_{2} & \mathrm{BaO} & \mathrm{La}_{2} \mathrm{O}_{3} & \mathrm{Ce}_{2} \mathrm{O}_{3} & \mathrm{Pr}_{2} \mathrm{O}_{3} & \mathrm{Nd}_{2} \mathrm{O}_{3} & \mathrm{Pm}_{2} \mathrm{O}_{3} \\ \mathrm{Sm}_{2} \mathrm{O}_{3} & \mathrm{Eu}_{2} \mathrm{O}_{3} & \mathrm{Gd}_{2} \mathrm{O}_{3} & \mathrm{~Tb}_{2} \mathrm{O}_{3} & \mathrm{Dr}_{2} \mathrm{O}_{3} & \mathrm{Ho}_{2} \mathrm{O}_{3} & \mathrm{Er}_{2} \mathrm{O}_{3} \\ \mathrm{Tm}_{2} \mathrm{O}_{3} & \mathrm{Yb}_{2} \mathrm{O}_{3} & \mathrm{Lu}_{2} \mathrm{O}_{3} & \mathrm{HFO}_{2} & \mathrm{Ta}_{2} \mathrm{O}_{5} & \mathrm{WO}_{3} & \mathrm{PbO} \\ \mathrm{ThO}_{2} & \mathrm{UO}_{2} & & & & & \end{array}$

tal forma que a cada novo ciclo, os fatores $\beta$ e as respectivas frações em peso dos óxidos são reavaliados, de acordo com o seguinte procedimento:

$$
\begin{aligned}
& \text { a. } \quad \beta_{i, j}=\frac{\sum_{j=1}^{n} \omega_{j} \cdot \alpha_{j}^{i}}{\sum_{j=1}^{n} \omega} \\
& \text { b. } \quad \omega_{i}=\omega_{j} \cdot \beta_{j} \\
& \text { c. } \omega_{j}=\omega_{i} \\
& \text { a1. }, \text { b1., etc } \quad 1 \leq i \leq n
\end{aligned}
$$

em que:

$$
\begin{aligned}
& \omega_{j}=\text { fração em peso para óxido j; } \\
& \omega_{i}=\text { fração em peso reavaliada para óxido j (na segunda, terceira, etc. }
\end{aligned}
$$

aproximações). 
Segue como na equação (6). $\beta_{\mathrm{ij}}$ em a refere-se ao valor do padrão, fornecido na opção (3). Na opção (2), o primeiro ciclo é iniciado em b.

Quando as frações obtidas em primeira aproximação são de boa qualidade, duas ou três iterações são suficientes; no caso de serem realizadas cinco iterações sem que haja convergência adequada dos dados, isto é, os resultados obtidos para cada fração nas duas últimas iterações divergem em valores superiores a $0,05 \%$, o procedimento é interrompido.

Os resultados progressivos de cada iteração (fatores $\beta$ e frações em peso de óxidos) são encaminhados à impressora; após a última iteração (bem sucedida), os resultados são expressos, adicionalmente, em proporção moleculares, de oxigênio e número de cátions por fórmula.

\section{ORGANIZAÇÃO DE ARQUIVOS: O PROGRAMA REORG ARQUI}

O programa REORG ARQUI foi incluido no conjunto para permitir ao usuário o manuseio com os arquivos de dados criados, de modo a possibilitar correções, supressões ou inclusões. É um programa auto-explicativo, de utilização muito simples, e que pode realizar as seguintes funções (v. detalhes sobre arquivos de dados em VLACH, 1988):

a. listar arquivos presentes em disquetes;

b. listar o conteúdo discriminado de arquivos de dados;

c. eliminar e criar arquivos;

d. subtrair, inserir ou corrigir em parte ou no todo o conteúdo dos registros de dados dos arquivos.

\section{UM EXEMPLO DE APUCAÇÃO}

Dados microanaliticos obtidos para anfibólios de rochas granitóides da Região de Morungaba (SP) são parcialmente reproduzidos nos Apêndices que seguem. Os padrões utilizados nas rotinas analiticas correspondem aos anfibólios Wilberforce e Kakanui, tal como registrados no Laboratório.

O Apêndice 1 reproduz parte de uma listagem de uma rotina analítica para $\mathrm{Fe}$, Mn e Mg efetuada com o programa ANLS ROTINA. Os dados apresentados foram adquiridos e armazenados com este programa e após recuperados e listados com REORG ARQUI. Constam desta 
listagem os valores obtidos para contantes de regressão linear para as variações do número de contagens por elemento em função de variações das correntes amostras, especificaçőes do material analisado (padrōes, amostras), tftulos para padrões $\theta / o u$ códigos de "pontos" analisados em cada amostra, o número de contagens obtido para cada um destes elementos e respectivas correntes de amostra. O número do registro de dados em que cada grupo de informaçōes está armazenado também é fornecido para facilitar o manuselo com estes arquivos. Os mesmos dados, porém com formato distinto, podem ser listados diretamente a partir de ANLS ROTINA.

No Apêndice 2 apresentam-se os dados convertidos para óxidos (em primeira aproximação) após correção para radlação de fundo e derlva $\theta$ transformação para porcentagens em peso dos óxidos por comparação com os padróes acima mencionados. No caso apresentado, foi selecionada a opção 2 do programa CORR DRIFT. Resultados finais para o anfibólio ANFI 238-A (hornblenda), considerando-se - além de $\mathrm{FeO}, \mathrm{MnO}$ e $\mathrm{MgO}-\mathrm{SiO}_{2}, \mathrm{TiO}_{2}, \mathrm{Al}_{2} \mathrm{O}_{3}, \mathrm{CaO}, \mathrm{Na}_{2} \mathrm{O}$ e $\mathrm{K}_{2} \mathrm{O}$, conjuntamente corrigidos para os efeitos de matriz com o programa CORREC BETA, são apresentados no Apêndice 3. Após três iterações, listam-se os resultados finais expressos em porcentagens em peso de óxidos, proporções moleculares e de oxigênio e respectivos números catiônicos.

\section{CONSIDERAÇÕES FINAIS}

O conjunto de programas apresentado é de grande auxilio para o procedimento microanalftico do Laboratório e, apesar das limitações do "hardware" instalado, que torna os programas algo vagarosos no tratamento de arquivos longos, o tempo médio gasto, quando comparado ao procedimento tradicional, é muito menor. Para materiais em que o fator fluorescência secundária é predominante dentre os efeitos de matriz e, portanto, a "correção beta" é pouco adequada, existe a possibilidade de utillzação da correção ZAF (cf. GOLDSTEIN et al., 1981), instalada no sistema EDS-LINK do Laboratório; porém, face às incompatibilidades de "hardware", os dados devem ser fornecidos manualmente. Felizmente, não é o caso da grande maioria dos materiais lá analisados.

\section{AGRADECIMENTOS}


São devidos ao Dr. G.Amaral, pelas "dicas" fornecidas na área de Informática, à Srta. I.Sonoki, pela colaboração dedicada ao programa CORREC BETA e ao Dr. E.Ruberti, pela leitura crítica do manuscrito. Este trabalho foi em parte realizado com auxilio FINEP/USP ( ${ }^{2}$ 4.2.86.0491.00, benificiário H.Ulbrich).

\section{REFERÊNCIAS BIBUOGRÁFICAS}

ALBEE, A.L. \& RAY, L (1970) Correction factors for electron-probe microanalysis of silicates, oxides, carbonates, phosphates and sulphates. Analytical Chemistry, 42: 14081414.

BENCE, A.E. \& ALBEE, A.L (1968) Empirical correction factors for the electron microanalysis of silicates and oxides. Journal of Geology, 76:382-403.

GOLDSTEIN, J.I. ; NEWBURY, D.E.; ECHUN, P.; JOY, D.C.; FIORI, C. \& LIFCHIN, E. (1981) Scanning electron microscopy and $\mathrm{X}$-ray microanalysis: a text for biologist, materials scientist and geologists. New York, Plenum Press. 673 p.

GOMES, C.B. (1984) Microssonda eletrônica: princípios e aplicações na geologia. In : GOMES. C.B. (ed.). Técnicas analfticas aplicadas à geologia. São Paulo, Edgar Blücher/Pró-Minério. p.159-208.

HEINRICH, K.F.J. (1981) Electron beam X-ray microanalysis. New York, Van Nostrand Teinhold Company.

KEIL, K. (1967) The electron microprobe X-ray analyser and its application in mineralogy. Fortschritte Mineralogie, 44(1):4-66.

VLACH, S.R.F. (1988) ESPERTO. Manual do usuário. 24 p. (inédito).

ZIEBOLD, T.O. \& OGILVIE, R.E. (1964) An empirical method for electron microanalysis. Analytical Chemistry, 36:322-327. 
Apêndice 1 - Listagens de dados "crus" obtidos no procedimento de aquisição de dados para os elementos Fe, Mn e Mg em anfibólios, através do programa ANLS ROTINA (v. texto).

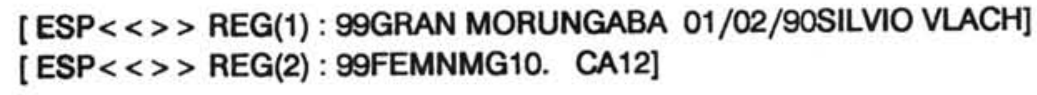

CR AMOSTRA COD/LOC COTG(FE) COTG(MN) COTG(MG) CORRENTE REG

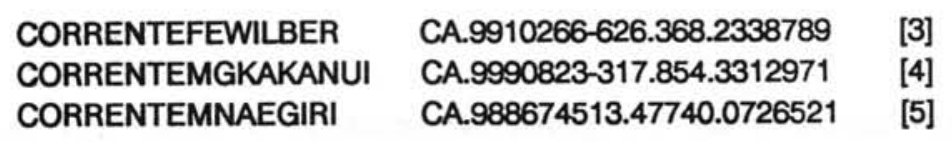

\begin{tabular}{|c|c|c|c|c|c|c|c|}
\hline & & & $\begin{array}{l}{[6]} \\
{[7]}\end{array}$ & & & & \\
\hline 01 & BACKGR & WILBER & 00106 & 00139 & 00145 & 26066 & 8 \\
\hline 01 & BACKGR & KAKANUI & 00097 & 00126 & 00145 & 26188 & 9 \\
\hline 01 & BACKGR & AEGIRI & 00107 & 00152 & 00146 & 26220 & 10 \\
\hline 01 & BACKGR & ANFI & 00103 & 00135 & 00152 & 26217 & 11 \\
\hline$\infty$ & BAKGR & ANFI & 00093 & 00171 & 00257 & 26195 & 12 \\
\hline$\infty$ & BAKGR & AEGIRI & 00096 & 00178 & 00227 & 26406 & 13 \\
\hline$\infty$ & BACKGR & KAKANUI & 00082 & 00152 & 00243 & 26323 & 14 \\
\hline$\infty$ & BACKGR & WILBER & 00101 & 00173 & 00261 & 26457 & 15 \\
\hline 10 & PADRAO & AEGIRI & 06991 & 01683 & 00821 & 26212 & 16 \\
\hline 10 & PADRAO & KAKANUI & 02710 & 00197 & 08296 & 26229 & 17 \\
\hline 10 & PADRAO & WILBER & 05610 & 00542 & 05249 & 26117 & 18 \\
\hline 11 & A-238-A & ANFI5B2 & 04432 & 00581 & 07571 & 26317 & 19 \\
\hline 11 & A-238-A & ANDFI5B1 & 03505 & 00626 & 09871 & 26300 & 20 \\
\hline 11 & A-238-A & ANFI5B3 & 04668 & 00739 & 06994 & 26122 & 21 \\
\hline 11 & A-238-A & ANFI5B4 & 04589 & 00636 & 07131 & 26148 & 22 \\
\hline 11 & A-238-A & ANFI5B5 & 04516 & 00646 & 06920 & 26110 & 23 \\
\hline 11 & A-238-A & ANFI5N1 & 04146 & 00664 & 08059 & 26116 & 24 \\
\hline 11 & A-238-A & ANF15N2 & 03839 & 00615 & 08794 & 25980 & 25 \\
\hline 11 & A-238-A & ANFI5N2 & 03485 & 00645 & 09726 & 26320 & 26 \\
\hline 11 & A-238-A & ANF15N3 & 03902 & 00651 & 08869 & 26256 & 27 \\
\hline 11 & A-238-A & ANFI4B1 & 04484 & 00618 & 07408 & 26303 & 28 \\
\hline 11 & A-238-A & ANF14B2 & 04684 & 00587 & 06851 & 26147 & 29 \\
\hline 11 & A-238-A & ANFI4B2 & 04667 & 00621 & 06781 & 26086 & 30 \\
\hline 11 & A-238-A & ANFI4B3 & 04531 & 00592 & 07226 & 26140 & 31 \\
\hline 11 & A-238-A & ANFI4B4 & 04516 & 00607 & 07189 & 26125 & 32 \\
\hline 11 & A-238-A & ANFI4N1 & 04425 & 00589 & 07251 & 26139 & 33 \\
\hline 11 & A-238-A & ANFI4N2 & 04441 & 00587 & 07349 & 26126 & 34 \\
\hline 11 & A-238-A & ANFI4N3 & 04494 & 00581 & 07312 & 26138 & 35 \\
\hline
\end{tabular}


Apêndice 2 - Listagens de dados corrigidos em primeira aproximação a partir dos valores "crus" apresentados no Apêndice 1 (v. texto).

99GRAN MORUNGABA 01/02/90SILVIO VLACH

99FEMNMG10. CA12

\# CORREÇAO DE DRIFT \#\#\# METODO: 2 \#

\begin{tabular}{|c|c|c|c|c|}
\hline AMOSTRA & COD/LOC & OX(FE) & OX(MN) & OX(MG) \\
\hline A-238-A & ANF15B2 & 16.42 & 1.204 & 11.44 \\
\hline A-238-A & ANF15B1 & 12.92 & 1.332 & 15.03 \\
\hline A-238-A & ANF15B3 & 17.46 & 1.667 & 10.63 \\
\hline A-238-A & ANF15B4 & 17.14 & 1.372 & 10.83 \\
\hline A-238-A & ANF15B5 & 16.89 & 1.403 & 10.52 \\
\hline A-238-A & ANF15N1 & 15.47 & 1.454 & 12.30 \\
\hline A-238-A & ANF15N2 & 14.38 & 1.325 & 13.53 \\
\hline A-238-A & ANF15N2 & 12.83 & 1.384 & 14.79 \\
\hline A-238-A & ANFI5N3 & 14.45 & 1.351 & 13.49 \\
\hline A-238-A & ANF14B1 & 16.63 & 1.309 & 11.20 \\
\hline A-238-A & ANFI4B2 & 17.50 & 1.233 & 10.40 \\
\hline A-238-A & ANFI4B2 & 17.48 & 1.334 & 10.31 \\
\hline A-238-A & ANF14B3 & 16.92 & 1.248 & 10.99 \\
\hline A-238-A & ANFI4B4 & 16.88 & 1.291 & 10.93 \\
\hline A-238-A & ANFI4N1 & 16.52 & 1.239 & 11.02 \\
\hline A-238-A & ANF14N2 & 16.59 & 1.234 & 11.18 \\
\hline A-238-A & ANF14N3 & 16.78 & 1.216 & 11.12 \\
\hline A-238-A & ANFI3I1 & 19.29 & .7303 & 10.77 \\
\hline A-238-A & ANFI3I2 & 18.87 & .7331 & 11.05 \\
\hline A-238-A & ANFI2B1 & 17.13 & 1.610 & 11.10 \\
\hline A-238-A & ANFI2B1 & 17.02 & 1.394 & 10.61 \\
\hline A-238-A & ANFI3B2 & 17.38 & 1.684 & 10.67 \\
\hline A-238-A & ANFI3B3 & 17.12 & 1.512 & 19.68 \\
\hline A-238-A & ANFI3B4 & 17.09 & 1.470 & 10.96 \\
\hline A-238-A & ANFI3N1 & 16.42 & 1.454 & 10.86 \\
\hline A-238-A & ANFI3N2 & 16.80 & 1.477 & 10.79 \\
\hline A-238-A & ANFI1B2 & 16.70 & 1.652 & 11.04 \\
\hline A-238-A & ANFI1B1 & 17.25 & 1.665 & 10.66 \\
\hline A-238-A & ANFI1N1 & 16.26 & 1.635 & 11.40 \\
\hline A-238-A & ANFI1N2 & 16.68 & 1.810 & 11.52 \\
\hline . & . & . & . & . \\
\hline . & . & . & . & . \\
\hline . & . & . & . & . \\
\hline
\end{tabular}


Apêndice 3 - Listagens dos resultados finais para $\mathrm{SiO}_{2}, \mathrm{TiO}_{2}, \mathrm{Al}_{2} \mathrm{O}_{3}, \mathrm{FeO}, \mathrm{MnO}, \mathrm{MgO}, \mathrm{CaO}, \mathrm{Na}_{2} \mathrm{O}$ e $\mathrm{K}_{2} \mathrm{O}$ do anfibólio ANFN1238-A, após as correções para o efeito matriz (v. texto).

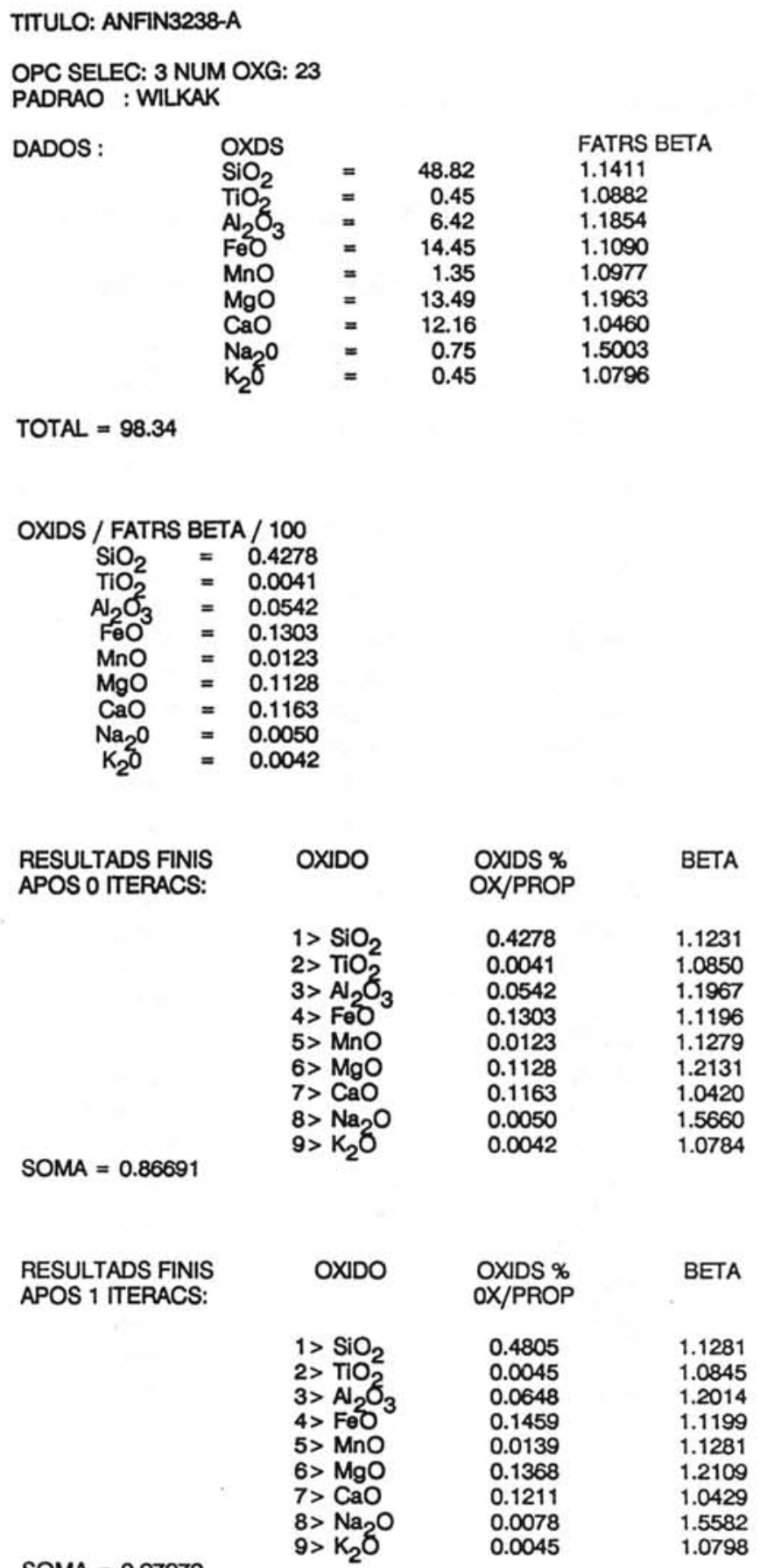




\begin{tabular}{|c|c|c|c|}
\hline $\begin{array}{l}\text { RESULTADS FINIS } \\
\text { APOS } 2 \text { ITERACS: }\end{array}$ & OXIDO & $\begin{array}{l}\text { OXIDS \% } \\
\text { OX/PROP }\end{array}$ & BETA \\
\hline SOMA $=0.98208$ & $\begin{array}{l}1>\mathrm{SiO}_{2} \\
2>\mathrm{TiO}_{2} \\
3>\mathrm{N}_{2} \mathrm{O}_{3} \\
4>\mathrm{FeO} \\
5>\mathrm{MnO} \\
6>\mathrm{MgO} \\
7>\mathrm{CaO} \\
8>\mathrm{Na}_{2} \mathrm{O} \\
9>\mathrm{K}_{2} \mathrm{O}\end{array}$ & $\begin{array}{l}0.4827 \\
0.0045 \\
0.0651 \\
0.1459 \\
0.0139 \\
0.1365 \\
0.1212 \\
0.0078 \\
0.0045\end{array}$ & $\begin{array}{l}1.1279 \\
1.0846 \\
1.2009 \\
1.1199 \\
1.1281 \\
1.2106 \\
1.0430 \\
1.5578 \\
1.0799\end{array}$ \\
\hline \multirow[t]{2}{*}{$\begin{array}{l}\text { RESULTADS FINIS } \\
\text { APOS } 3 \text { ITERACS: }\end{array}$} & OXIDO & $\begin{array}{l}\text { OXIDS \% } \\
\text { OX/PROP }\end{array}$ & BETA \\
\hline & $\begin{array}{l}1>\mathrm{SiO}_{2} \\
2>\mathrm{TiO}_{2} \\
3>\mathrm{N}_{2} \mathrm{O}_{3} \\
4>\mathrm{FeO} \\
5>\mathrm{MnO} \\
6>\mathrm{MgO} \\
7>\mathrm{CaO} \\
8>\mathrm{Na}_{2} \mathrm{O} \\
9>\mathrm{K}_{2} \mathrm{O}\end{array}$ & $\begin{array}{l}0.4825 \\
0.0045 \\
0.0650 \\
0.1459 \\
0.0139 \\
0.1365 \\
0.1212 \\
0.0078 \\
0.0045\end{array}$ & $\begin{array}{l}1.1279 \\
1.0846 \\
1.2009 \\
1.1199 \\
1.1281 \\
1.2107 \\
1.0430 \\
1.5579 \\
1.0799\end{array}$ \\
\hline
\end{tabular}

SOMA $=0.98191$

OXIDS

\% PESO

PROPORC.

PROPORC. MOLECULAR

OXIGENIO

NUMERO

0.80309
0.00561
0.06379
0.20311
0.01956
0.33866
0.21621
0.01256
0.00478

1.60618

0.01123

0.19136

0.20311

0.01956

0.33866

0.21621

0.01256

0.7787

$\mathrm{Na}_{2} \mathrm{O}$

0.4501

0.00478

CATIONICO

FATOR P/ CONVERS. OXG -> 23 / $8.83376: 2.60365$ 